\title{
Diverse Roles of JNK and MKK Pathways in the Brain
}

\author{
Tokiwa Yamasaki, ${ }^{1}$ Hiroshi Kawasaki, ${ }^{2,3}$ and Hiroshi Nishina ${ }^{1}$ \\ ${ }^{1}$ Department of Developmental and Regenerative Biology, Medical Research Institute, Tokyo Medical and Dental University, \\ Tokyo 113-8510, Japan \\ ${ }^{2}$ Department of Molecular and Systems Neurobiology, Graduate School of Medicine, University of Tokyo, 7-3-1 Hongo, Bunkyo-ku, \\ Tokyo 113-0033, Japan \\ ${ }^{3}$ Global COE Program "Comprehensive Center of Education and Research for Chemical Biology of the Diseases", University of Tokyo, \\ Tokyo 113-0033, Japan
}

Correspondence should be addressed to Hiroshi Nishina, nishina.dbio@mri.tmd.ac.jp

Received 11 August 2011; Accepted 1 November 2011

Academic Editor: M. Gaestel

Copyright ( $\odot 2012$ Tokiwa Yamasaki et al. This is an open access article distributed under the Creative Commons Attribution License, which permits unrestricted use, distribution, and reproduction in any medium, provided the original work is properly cited.

The c-Jun $\mathrm{NH}_{2}$-terminal protein kinase (JNK) plays important roles in a broad range of physiological processes. JNK is controlled by two upstream regulators, mitogen-activated protein kinase kinase (MKK) 4 and MKK7, which are activated by various MAPKKKs. Studies employing knockout mice have demonstrated that the JNK signaling pathway is involved in diverse phenomena in the brain, regulating brain development and maintenance as well as animal metabolism and behavior. Furthermore, examination of single or combined knockout mice of $J n k 1$, Jnk2, and $J n k 3$ has revealed both functional differences and redundancy among JNK1, JNK2, and JNK3. Phenotypic differences between knockouts of MKK4 and MKK7 have also been observed, suggesting that the JNK signaling pathway in the brain has a complex nature and is intricately regulated. This paper summarizes the functional properties of the major JNK signaling components in the developing and adult brain.

\section{Introduction}

The c-Jun $\mathrm{NH}_{2}$-terminal protein kinases (JNKs, also called stress-activated protein kinases (SAPKs)) are members of the evolutionarily conserved mitogen-activated protein kinase (MAPK) family $[1,2]$. The JNK subfamily consists of three related genes: Jnk1, Jnk2, and Jnk3. In mammals, the JNK1 and JNK2 proteins are ubiquitously expressed, whereas JNK3 is found almost exclusively in the brain and testis. JNKs are activated by many types of external stress, including heat shock, UV irradiation, and inflammatory cytokines. JNKs phosphorylate numerous important substrates, including the transcription factors AP-1 and c-Jun, various apoptotic proteins, and microtubule-associated proteins (MAPs) [37]. Through phosphorylation of these substrates, JNKs regulate gene expression governing stress responses as well as the normal physiological processes of cell proliferation, apoptosis, differentiation, and cell migration.

Activation of JNK is catalyzed by two kinases, mitogenactivated protein kinase kinase (MKK) 4 and MKK7 [810]. Although MKK4 and MKK7 are both dual-specificity
Thr and Tyr kinases, previous studies of JNK activation have shown that MKK4 preferentially phosphorylates the Tyr residue of the TPY motif in the activation loop of JNKs, whereas MKK7 preferentially phosphorylates the Thr residue $[11,12]$. The activation of MKK4 and MKK7 is mediated by various MAPKKKs, including mixed lineage protein kinases (MLKs), apoptosis signal-regulating kinases (ASKs) and dual leucine zipper kinase (DLK) [13]. In addition to regulation by these upstream kinases, the JNK signaling pathway is modulated by various scaffold proteins, including JNKinteracting protein (JIP) 1, JIP2, and JIP3 (also known as JNK/SAPK associated protein-1 (JSAP1)) [14-17]. These scaffold proteins assemble multienzyme complexes that involve a specific triad of a MAPKKK, a MAPKK, and a MAPK and provide an insulated physical conduit for signal transduction. The resulting linkage of these kinases forms a functional signaling module that performs transduction duties for a particular purpose.

In most mammalian cell types, JNK activation is tightly controlled and employed at moderate levels in specific circumstances. In contrast, JNK signaling in the brain is 


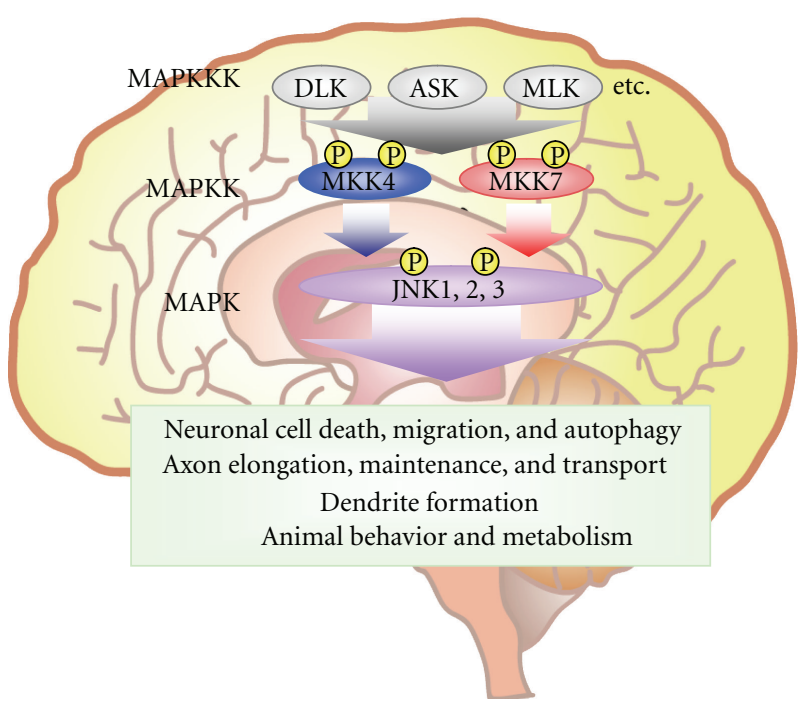

FIGURE 1: The JNK signaling pathway in the mammalian brain. A MAPKKK, such as DLK, ASK, or MLK, phosphorylates (P) and activates a MAPKK such as MKK4 or MKK7. An activated MAPKK in turn phosphorylates and activates a MAPK such as JNK1, JNK2, or JNK3. Activated JNKs then regulate various phenomena in the brain.

highly and constitutively activated. Studies of gene knockout (KO) mice lacking JNKs or their upstream kinases have shown that this sustained activation is necessary to fulfil the diverse and essential roles that JNK signaling plays in the brain (Figure 1). This paper summarizes the phenotypes of these mutant animals and discusses what they reveal about the regulation and various functions of JNK and MKK signaling in the brain.

\section{Brain Phenotypes of JNK Knockout Mice}

2.1. Jnk1/2 DKO Mice: JNK1/2 Induces Programmed Cell Death during Early Brain Development. Knockout mice lacking one, two, or all JNK isoforms have been generated over the past decade, and their diverse phenotypes have been reported (Table 1). Importantly, embryonic lethality is not caused by the single $\mathrm{KO}$ of any Jnk gene. Two strains of double KO mice, Jnk1 $1^{-/-} J n k 3^{-/-}(J n k 1 / 3$ DKO) and $J n k 2^{-/-} J n k 3^{-/-}(J n k 2 / 3 \mathrm{DKO})$, are viable, but Jnk1 ${ }^{-/-} J n k 2^{-/-}$ (Jnk1/2 DKO) animals exhibit early embryonic lethality.

During early brain development in mice, the neural folds emerge at embryonic day (E) 7.5 in the cephalic region, close over at E8.5-E9 (neural tube closure), and form the neural tube. The Jnk1/2 DKO mouse is lethal at E11.5 due to dysregulation of apoptosis in the neural tube $[18,19]$. Specifically, there is a marked reduction in cell death in the lateral edges of the hindbrain prior to neural tube closure. In contrast, increased apoptosis and caspase activation are found in the mutant forebrain, leading to precocious degeneration. Interestingly, about $25 \%$ of $J n k 1^{-/-} J n k 2^{+/-}$fetuses display excencephaly that is likely the result of failed neural tube closure, whereas $J n k 1^{+/-} J n k 2^{-/-}$ mice are normal [19]. These results suggest that JNK1 and
TABLE 1: Phenotypes of JNK knockout mice.

\begin{tabular}{|c|c|c|}
\hline Mouse model & Phenotypes & References \\
\hline$J n k 1^{-1-}$ & $\begin{array}{l}\text { Accelerated radial migration } \\
\text { Shorter dendrites with increased } \\
\text { branching } \\
\text { Degeneration of anterior } \\
\text { commissure } \\
\text { Enhanced glucocorticoid- or } \\
\text { insulin-induced food intake }\end{array}$ & $\begin{array}{c}{[20-} \\
22,31]\end{array}$ \\
\hline$J n k 2^{-/-}$ & $\begin{array}{l}\text { Resistance to MPTP-induced } \\
\text { neuronal cell death }\end{array}$ & {$[24]$} \\
\hline$J n k 3^{-/-}$ & $\begin{array}{l}\text { Resistance to MPTP-induced } \\
\text { neuronal cell death } \\
\text { Resistance to kainic acid-induced } \\
\text { neuronal cell death } \\
\text { Resistance to ischemia-induced } \\
\text { neuronal apoptosis } \\
\text { Resistance to } \\
\text { 6-hydrozydopamine-induced } \\
\text { neuronal apoptosis }\end{array}$ & $\begin{array}{c}{[24-} \\
27,29]\end{array}$ \\
\hline$J n k 1^{-/-} J n k 2^{-/-}$ & $\begin{array}{l}\text { Embryonic lethal at E11.5 } \\
\text { Defective neural tube closure } \\
\text { Dysregulated apoptosis in brain } \\
\text { (increased in forebrain, reduced } \\
\text { in hindbrain) }\end{array}$ & {$[18,19]$} \\
\hline$J n k 2^{-/-} J n k 3^{-/-}$ & $\begin{array}{l}\text { Resistance to MPTP-induced } \\
\text { neuronal cell death }\end{array}$ & {$[24]$} \\
\hline $\begin{array}{l}\text { Jnk } 1^{\text {flox/flox }} \\
\text { Nestin-Cre }\end{array}$ & $\begin{array}{l}\text { Animal protected from } \\
\text { diet-induced glucose intolerance } \\
\text { and insulin resistance } \\
\text { Reduced serum IGF-1 and GH } \\
\text { Increased serum thyroid } \\
\text { hormones }\end{array}$ & {$[30]$} \\
\hline 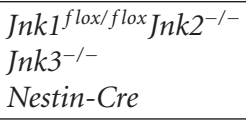 & Early embryonic lethal & {$[53]$} \\
\hline $\begin{array}{l}n k 1^{\text {flox/flox } J n k 2^{-/-}} \\
\text {Jnk3 } 3^{-/-} \\
\text {Pcp2-Cre }\end{array}$ & $\begin{array}{l}\text { Loss of dendritic arborization } \\
\text { Axon hypertrophy } \\
\text { Increased autophagic vacuoles }\end{array}$ & {$[53]$} \\
\hline
\end{tabular}

JNK2 are redundant regulators of programmed cell death during early brain development and that JNK1 is dominant in this particular function of the JNK signaling pathway.

\subsection{Jnk1 ${ }^{-/-}$Mice: JNK1 Is Required for Neuronal Migration,} Dendrite Formation, and Axon Maintenance during Later Brain Development. After neural tube formation at E8.5-E9, three of its vesicles give rise to various specialized regions of the brain. Neuronal progenitors proliferate and generate immature neurons. These cells subsequently migrate from the proliferative zones to their final positions, where they extend neurites. For example, in the developing cortex, neural progenitors proliferate from E11 to E17 then generate immature neurons that migrate and form the cortical layers, the cortical plate, subplate, intermediate zone, and ventricular zone. The extension and maturation of neurites by these cells then continues during the remaining embryonic and postnatal stages. Jnk1 $1^{-/-}$embryos display thicker cortical 
plates and thinner ventricular zones than wild-type animals [20]. Jnk1 $1^{-1-}$ embryos also show abnormally accelerated migration of cortical neurons (radial migration), a process whose precise regulation is required for the correct formation of cortical layers. These findings indicate that JNK1 regulates the rate of neuronal migration during cortical development.

Other studies have shown that JNK1 also regulates neurite formation and maintenance. Neurons extend two types of neurites: dendrites and axons. Compared to wildtype mice, dendrites in the cortex and cerebellum of $J n k 1^{-1-}$ mice are shorter and have more processes [21], indicating that JNK1 plays an important role in defining dendritic architecture during brain development. In contrast, axon formation is not affected by JNK1 disruption. Axon tracts such as the corpus callosum and anterior commissure appear normal in $J n k 1^{-/-}$mice until postnatal day (P) 6. From P6$\mathrm{P} 12$, however, the anterior commissure degenerates, demonstrating that JNK1 is required for axon maintenance [22].

One of the molecular mechanisms mediating JNK pathway functions during brain development and maintenance is the phosphorylation of MAPs such as MAP1B, MAP2, and superior cervical ganglion 10 (SCG10) [20-22]. MAPs bind to microtubules (MTs) and modulate their stability and structure. Importantly, the phosphorylation of MAPs by one of several protein kinases (including JNK) regulates the binding of these enzymes to MTs and thus the regulation of MT modification. In $J n k 1^{-/-}$brain, the phosphorylation of MAP1B, MAP2, and SCG10 is reduced. Moreover, the introduction of a mutated SCG10 protein that mimics the JNK1-phosphorylated form restores normal neuronal migration in the brains of $J n k 1^{-/-}$embryos [20]. These results demonstrate that, in addition to regulating radial migration, dendrite formation, and axon maintenance in the developing brain, JNK1 controls MT structure through MAP phosphorylation.

2.3. Jnk2 $2^{-/-}$and Jnk3 $3^{-/-}$Mice: JNK2 and/or JNK3 Are Required for Neuronal Cell Death Induced by Neuronal Stresses in Adult Brain. The redundancy of many JNK functions is highlighted by the fact that $J n k 2^{-/-}$and $J n k 3^{-/-}$ single $\mathrm{KO}$ mice show none of the defects observed in $J n k 1^{-/-}$mice. However, Jnk2 $2^{-/-}$and $J n k 3^{-/-}$mice do show alterations in neuronal stress-induced neuronal cell death that are rarely observed in $J n k 1^{-/-}$mice, implying that each JNK enzyme has a unique function. For example, 1-methyl-4-phenyl-1,2,3,6-tetrahydropyridine (MPTP) is a neurotoxin whose administration induces dopaminergic cell demise and so results in symptoms that replicate most of the neuropathological hallmarks of Parkinson's disease

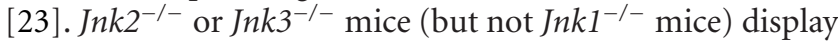
resistance to MPTP-induced neuronal cell death in vivo [24]. Moreover, this resistance to MPTP is enhanced by double mutation of Jnk2 and Jnk3, indicating that JNK2 and JNK3 play partially overlapping roles in this process. $J n k 3^{-/-}$mice also display resistance to kainic acid-induced cell death (excitotoxicity-induced apoptosis) and ischemia-induced cell death in vivo [25-27], as well as reduced susceptibility to apoptosis induced by the Alzheimer's disease-related protein beta-amyloid in vitro [28]. Finally, Jnk $3^{-/-}$mice intrastriatally injected with 6-hydroxydopamine, which provokes the death of dopaminergic neurons, show a transient prolongation of dopaminergic neuron survival in the substantia nigra compacta compared to injected control mice [29]. These results suggest that the physiological function of both JNK2 and JNK3 in the brain is to induce apoptosis in response to neuronal stress.

\subsection{Conditional Jnk1 $1^{-/-}$Mice: JNK1 Activity in Adult Brain} Regulates Animal Metabolism. Intact JNK signaling in the adult brain is required for normal animal metabolism. A high-fat diet induces JNK activation in the hypothalamus and pituitary, which regulate body weight control, glucose homeostasis, and secretion of hormones. Conditional KO (cKO) mice have been generated in which Jnk1 expression is controlled by Nestin-Cre, which induces Cre recombinase expression in neural stem cells [30]. Upon high-fat feeding, Jnk $1^{\text {flox/flox }}$ Nestin-Cre mice exhibit increased insulin sensitivity (compared to wild-type controls) both in the CNS and in peripheral tissues, improved glucose metabolism and protection from hepatic steatosis and adipose tissue dysfunction. Jnk $1^{\text {flox/flox }}$ Nestin-Cre mice also display reduced somatic growth and altered secretion of growth hormone $(\mathrm{GH})$, insulin-like growth factor (IGF), and thyroid hormones. JNK activity in the brain is thus required for normal metabolism.

JNK signaling in the brain is also involved in the control of feeding. The hypothalamus governs food intake in response to nutrient status, cytokines, and hormones. Jnk1 $1^{-I-}$ mice exhibit enhanced food intake and weight gain upon hypothalamic administration of glucocorticoid [31]. Moreover, JNK1 disruption increases mouse sensitivity to the anorexigenic effects of hypothalamic insulin administration. These data show that JNK signaling in the brain regulates not only hormone secretion and peripheral metabolism but also feeding and thus contributes to the maintenance of energy homeostasis. It remains unclear whether JNK2 and/or JNK3 are also involved in these functions, but improved insulin resistance has been reported for both $J n k 1^{+/-} J n k 2^{-/-}$mice and $J n k 1^{-1-}$ mice fed a high-fat diet $[32,33]$. These results suggest that JNK2 may be involved in the central and/or peripheral regulation of glucose homeostasis. Analyses of cKO mice lacking Jnk1 and/or Jnk2 will resolve this issue.

\section{Brain Phenotypes of MKK Knockout Mice}

Because JNK isoforms are required for brain development, and MKK4 and MKK7 activate all JNK isoforms, it is not surprising that disruption of $M k k 4$ and/or $M k k 7$ results in severe defects in mouse development. $M k k 4^{-/-} M k k 7^{-/-}$ $(M k k 4 / 7$ DKO) mice die at E8.5 before neural tube formation, and both $M k k 4^{-/-}$and $M k k 7^{-1-}$ single KO mice are embryonic lethal at around E11.5 due to impaired liver formation $[9,34-36]$. To circumvent this limitation, the functions of MKK4 and MKK7 during brain development have been investigated using Nestin-Cre cKO mice lacking MKK4 and/or MKK7. Analyses of these mutants have proven very helpful in overcoming the conundrum posed by the redundancy of JNK isoforms. 
3.1. Mkk4flox/flox Nestin-Cre Mice: MKK4 Is Required for Neuronal Migration and Axon Maintenance in the Developing Brain. In Mkk4 flox/flox Nestin-Cre mice, total JNK activation in the brain is reduced to $20 \%$ of normal but these animals are not embryonic lethal and dysregulated apoptosis is not observed [37]. At birth, Mkk4flox/flox Nestin-Cre mice are indistinguishable from their control littermates, but the mutants stop growing a few days later and die at around 3 weeks of age. The brains of Mkkfflox/flox Nestin-Cre mice display misaligned Purkinje cells in the cerebellum and delayed radial migration in the cerebral cortex. In their commissural axon tracts, axonal degeneration is observed not only in the anterior commissure, the site of a similar defect in $J n k 1^{-/-}$brain [22], but also in the corpus callosum. At the molecular level, hypophosphorylation of MAP1B is observed in Mkk4flox/flox Nestin-Cre mice, suggesting that dysregulation of MT dynamics is involved in these phenotypes. The altered neuronal cell migration and axon maintenance observed in Mkk4flox/flox Nestin-Cre mice are also seen in $J n k 1^{-/-}$mice, but these phenotypes are less severe in the latter. Thus, MKK4 is a regulator of radial migration and axon maintenance in the brain, and MKK4's effects are mediated not only by JNK1 but also by JNK2 and/or JNK3.

3.2. Mkk7flox/flox Nestin-Cre Mice: MKK7 Is Required for Neuronal Migration and Axon Elongation in Developing Brain. Our group has generated $M k k 7^{\text {flox } / \text { flox }}$ Nestin-Cre mice [38]. Unlike Mkk4flox/flox Nestin-Cre mice, which survive until age 3 weeks, Mkk7flox/flox Nestin-Cre mice die at birth without breathing. Like Mkk4flox/flox Nestin-Cre mice, JNK activation is reduced to $20 \%$ of normal in the developing brain of Mkk7flox/flox Nestin-Cre mutants, and a delay in neuronal migration in the cerebrum is observed. However, other phenotypes do not overlap between $M k k 7^{f l o x} / f l o x$ Nestin-Cre and Mkk4flox/flox Nestin-Cre mice (Table 2). At E18.5, Mkk7flox/flox Nestin-Cre mice display enlarged brain ventricles, diminished striatum, decreased forebrain axon tracts, and reduced corticofugal axons; none of these defects has been found in Mkk4flox/flox Nestin-Cre mice. In addition, ultrastructural alterations such as abnormal accumulations of filamentous structures and autophagic vacuoles are observed in Mkk $7^{\text {flox/flox }}$ Nestin-Cre brain but not in Mkk4flox/flox Nestin-Cre brain. Thus, Mkk7 has unique functions in the developing brain that differ from those of MKK4.

Differences between MKK7 and MKK4 functions also appear at the molecular level. In Mkk4 flox/flox Nestin-Cre brain, phosphorylation levels of MAP1B are reduced but DCX phosphorylation is not altered. In contrast, phosphorylation levels of both MAP1B and DCX are decreased in $M k k 7^{\text {flox/flox }}$ Nestin-Cre brain, suggesting that the MKK7JNK and MKK4-JNK signaling modules in this organ are not identical. In line with this hypothesis, the scaffold protein JIP1 binds to JNK, MKK7, and DCX but not to MKK4. We therefore propose that differences in scaffold proteins and/or substrates involved in the MKK7-JNK versus MKK4-JNK pathways could cause the phenotypic divergence observed

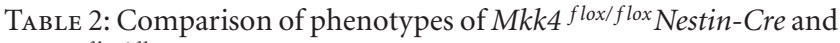
Mkk7 flox/flox Nestin-Cre mice.

\begin{tabular}{lcc}
\hline Phenotype & $\begin{array}{c}\text { Mkk4flox/flox } \\
\text { Nestin-Cre [37] }\end{array}$ & $\begin{array}{c}\text { Mkk flox/flox } \\
\text { Nestin-Cre [38] }\end{array}$ \\
\hline $\begin{array}{l}\text { Neuronal JNK activity } \\
\text { Age of lethality }\end{array}$ & Suppressed & Suppressed \\
Brain ventricle size at & Around 3 weeks old & At birth \\
E18.5 & Not reported & Enlarged \\
Striatum & Not reported & Reduced \\
Axon tracts (E18.5) & Unaffected & Greatly reduced \\
Axon tracts (postnatal) & Less fasciculated & Not determined \\
Intermediate filaments & Not reported & Accumulate in \\
Autophagic vacuoles & Not reported & Accumulate \\
JNK phosphorylation in & Not reported & Suppressed \\
axons & Unaffected & Reduced \\
L1-positive axons (E18.5) & Not reported & Reduced \\
TAG-1-positive axons & Unaffected & Unaffected \\
Apoptosis & Expressed \\
Cortical layer markers & Not reported & Delayed \\
Radial migration & Delayed & Suppressed \\
Phosphorylation of c-Jun & Suppressed & Suppressed \\
Phosphorylation of NF-H & Suppressed & Suppressed \\
Phosphorylation of & Suppressed & Suppressed \\
MAP1B & Unaffected &
\end{tabular}

between $M k k 7^{\text {flox/flox }}$ Nestin-Cre and MKK $4^{\text {flox/flox }}$ NestinCre mice (Figure 2).

\section{Brain Phenotypes of MAPKKK Knockout Mice}

Compared with MAPKKs, MAPKKKs comprise a much larger family of related proteins. Indeed, at least 12 MAPKKKs have been identified as regulating various steps of the JNK signaling pathway [13]. This multiplicity of related functions suggests that each MAPKKK has a specific spatiotemporal role in controlling JNK signaling. However, the precise mechanisms by which most MAPKKKs regulate JNKs and their biological roles in the brain have yet to be fully elucidated. We summarize below evidence supporting the importance of two MAPKKKs, DLK, and ASK1, in the JNK signaling pathway in the brain.

4.1. Dlk ${ }^{-/-}$Mice: DLK Is Required for Axon Elongation and Neuronal Migration in Developing Brain. DLK is known to be critical in the developing mammalian brain. $D l k^{-/-}$mice die perinatally, with no homozygous mutant surviving until weaning. $D l k^{-/-}$mice display retarded radial migration and impaired fiber tract development by neocortical pyramidal neurons in the cerebrum [40]. Direct and quantitative analysis of pyramidal neuron radial migration using slice culture and a time-lapse imaging system has revealed that $D l k$ 


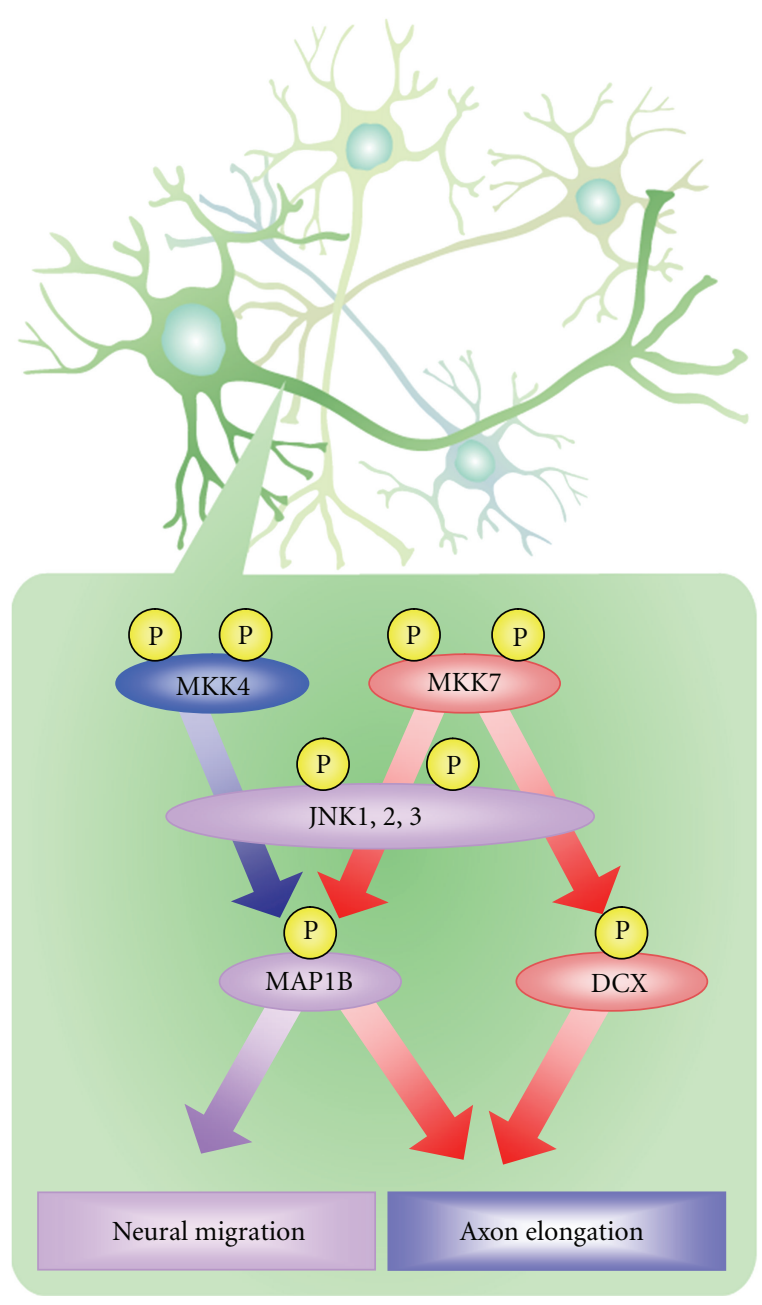

FIgURE 2: MKK4 and MKK7 have different functions in the developing brain. MKK4 and MKK7 both activate JNKs, which phosphorylate MT-associated proteins such as MAP1B and DCX. Activated MAP1B and DCX regulate neuronal migration and axon elongation in the developing brain. However, JNK activated by MKK4 regulates radial migration but not axon elongation, whereas JNK activated by MKK7 controls both radial migration and axon elongation. These differences between MKK7 and MKK4 functions also appear at the molecular level. The phosphorylation of MAP1B requires that JNK be activated by both MKK4 and MKK7. However, the phosphorylation of DCX requires JNK activation only by MKK7 and not by MKK4.

disruption affects acceleration around the cortical subplate. Furthermore, in vitro culture of $D l k^{-/-}$neurons has shown that DLK is involved in the establishment of neuronal polarity and regulates the MT dynamics driving the transition of stage 1 (nonpolar) neurons to stage 2 (multipolar) neurons and then the transition of stage 2 to stage 3 (axonforming) neurons [41]. These results demonstrate that DLK regulates radial migration and axon formation during brain development. However, a reduction of only $30 \%$ in total phosphorylated JNK is observed in $D l k^{-/-}$brain, indicating that other MAPKKKs must be involved in JNK activation in this organ. The identities of these enzymes are under active investigation.

4.2. Ask1 $1^{-1-}$ Mice: ASK1 Drives Neuronal Cell Death in Adult Brain Following Ischemia or Neurodegeneration and Regulates Animal Behavior. ASK1 is well known as a proapoptotic MAPKKK that is involved in responses to diverse stresses and activates both the JNK and p38 signaling pathways [42]. $A s k 1^{-/-}$mice are born at the expected Mendelian frequency and show no developmental abnormalities as determined by histological analysis [43]. Retinal ganglion cells of $A s k 1^{-/-}$ mice do not readily undergo ischemia-induced apoptosis in vivo [44], and $A s k 1^{-/-}$primary neurons display resistance to neuronal cell death triggered by polyglutamine in vitro [45]. In mice transgenic for a mutation of $\mathrm{Cu} / \mathrm{Zn}$-superoxide dismutase, which serve as a model of human amyotrophic lateral sclerosis, deletion of ASK1 mitigates motor neuron loss and extends mouse lifespan [46]. These data indicate that ASK1's proapoptotic functions extend to neurons in vivo. ASK1 also has nonapoptotic functions in the brain, since $A s k 1^{-1-}$ mice exhibit temporary hyperactivity in an openfield test [47]. Interestingly, this hyperactivity is specific to the novel environment, with $A s k 1^{-/-}$mice displaying normal activities in the familiar field. Ask1-/- mice also show impaired novelty preference at 24 hours after training but superior performance on the rotarod test. These results demonstrate that ASK1 is involved in locomotor activity, novelty preference, and motor coordination requiring dopaminergic transmission.

\section{Other Evidence Supporting Roles for JNK Signaling in the Nervous System}

5.1. Regulation of Axonal Transport. In Drosophila, an absence of the function of either Bsk (Drosophila JNK) or Hep (Drosophila MKK7) causes a failure of lateral epithelial cells to stretch such that the embryo develops a hole in the dorsal cuticle [48-50]. These data indicate that the MKK7JNK signaling pathway mediates cell migration in Drosophila and regulates dorsal closure during early morphogenesis.

Axonal transport in Drosophila is driven along MT by the kinesin motor system, and APLIP (Drosophila JIP1) is known as a "cargo linker" that joins kinesin-1 to various vesicle proteins such as the Drosophila equivalent of the Alzheimer's APP protein [51]. Drosophila axonal transport is believed to be regulated by the JNK signaling pathway because mutation of Wnd (Drosophila DLK), Hep, or Bsk results in the abnormal accumulation of synaptic vesicles in the nerves of third instar larvae [39]. Mutation of Wnd or Hep also disrupts the binding of kinesin-1 to APLIP1. Thus, the JNK signaling pathway regulates the attachment of APLIP1 to kinesin-1 in Drosophila, ensuring that the dissociation of kinesin-1 from its vesicle protein cargo occurs at the appropriate position (Figure $3(\mathrm{a})$ ).

The involvement of the JNK signaling pathway in axonal transport has also been reported in mammals. JNK can be activated by the pathogenic polyglutamine-containing Huntingtin protein associated with human Huntington's disease. Fast axonal transport is then inhibited because JNK3 


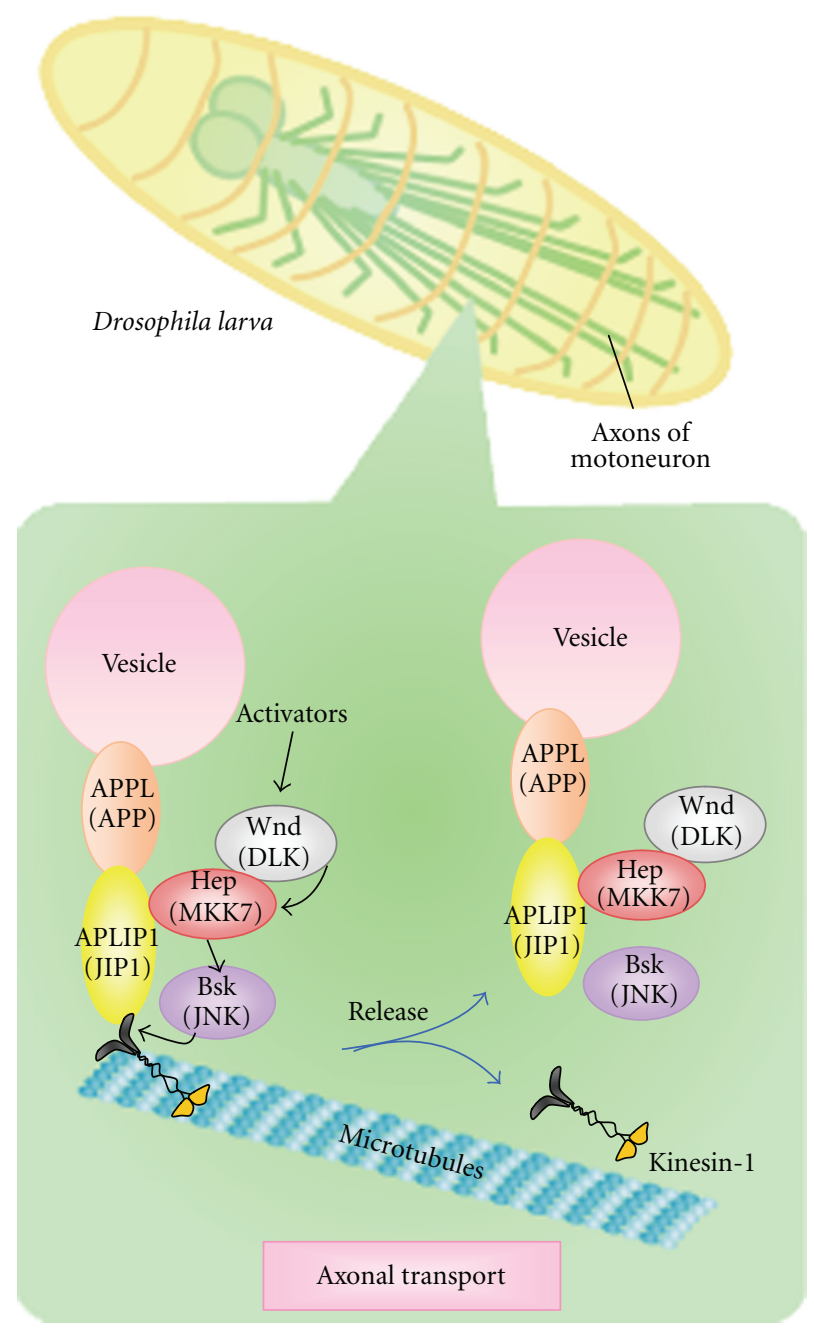

(a)

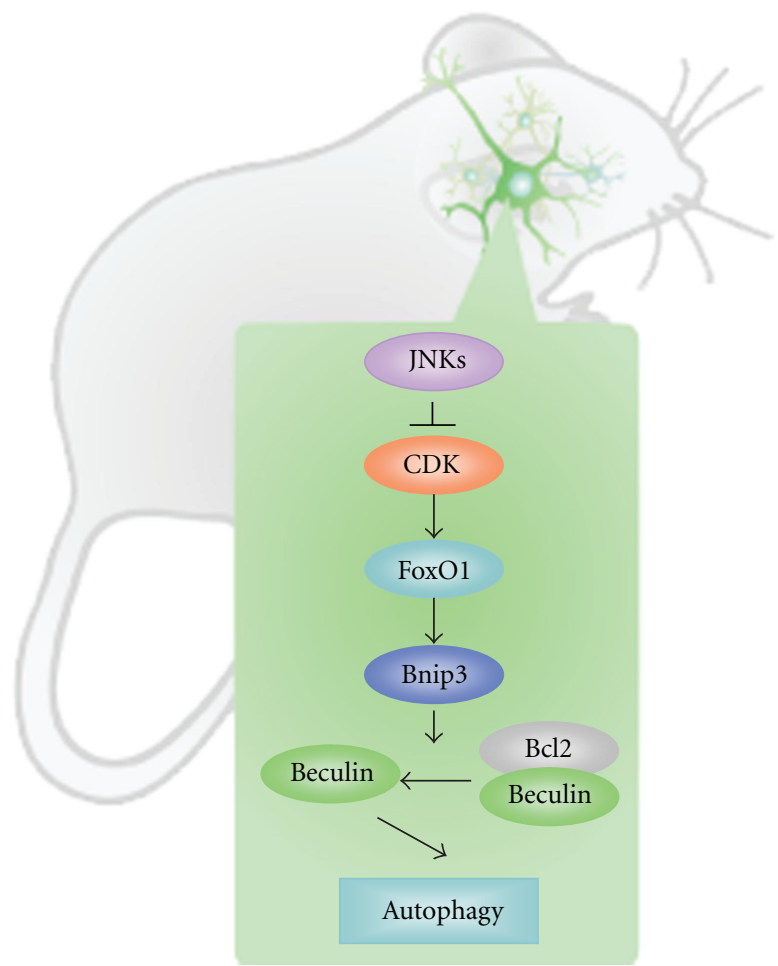

(b)

FIGURE 3: The JNK signaling pathway regulates axonal transport and autophagy. (a) Model of how the JNK signaling pathway controls axonal transport in Drosophila. Axonal transport is driven along MT by kinesin-1, which binds to the vesicles that make up its cargo through APLIP1 (Drosophila JIP1) and APPL (APP). This process is driven by Wnd (DLK), which is activated by unknown upstream signals, and phosphorylates Hep (MKK7). Activated Hep then phosphorylates and activates Bsk (JNK), which then directly or indirectly modifies the linkage complex and causes APLIP1 and the cargo to dissociate from kinesin-1. (This figure is excerpted from [39] with some modifications.) (b) Model of the regulation of neuronal autophagy in mice. In normal neurons, constitutively activated JNKs suppress CDK-induced FoxO1 activation, preventing autophagy. When all three of JNK1, JNK2, and JNK3 are disrupted, CDK-mediated FoxO1 activation increases Bnip3 expression. High levels of Bnip inhibit the binding of Beclin1 to Bcl2, and this freshly released Beclin1 induces autophagy.

directly phosphorylates the kinesin-1 motor domain [52]. It remains to be determined how extensively this molecular mechanism is conserved between Drosophila and mammals.

5.2. Regulation of Neuronal Autophagy. Conditional Jnkl, Jnk2, Jnk3 triple knockout mice (Jnk1/2/3 cTKO mice) have been generated to eliminate the problem of functional redundancy among JNK1, 2, and 3 [53]. These animals were created by crossing Jnk1 flox mice with $J n k 2^{-/-}, J n k 3^{-/-}$, and Cre-expressing strains. Jnk1/2/3 cTKO Nestin-Cre mice are embryonic lethal at an early stage, but $J n k 1 / 2 / 3$ cTKO Pcp2Cre mice, which express Cre in Purkinje cells, are viable.
While numerous abnormal phenotypes have been reported for Jnk1/2/3 cTKO Pcp2-Cre mice, including axon hypertrophy, abnormal mitochondrial transport, and prolonged cellular lifespan in culture, the most striking anomaly observed has been increased neuronal autophagy [53]. Results to date indicate that, in neurons, the JNK signaling pathway suppresses autophagy, whereas, in nonneuronal cells, JNK signaling either induces autophagy or serves as an effector of autophagy-associated cell death [54, 55]. Although the JNK substrate leading to autophagy has yet to be identified, it has been shown that increased autophagy in Jnk1/2/3 cTKO neurons is mediated by FoxO1 and not by an mTORC1dependent mechanism [53]. The combined disruption of 
JNK1, JNK2, and JNK3 increases Bnip3 expression through FoxO1 activation that is mediated by CDK (Figure 3(b)). Bnip3 inhibits Beclin1-Bcl2 binding and induces Beclin1 release, which in turn triggers autophagy.

\section{Conclusion and Future Perspectives}

Studies of KO mice lacking JNKs, MAPKKs or MAPKKKs, have revealed that the JNK signaling pathway is involved in diverse roles in the brain, including induction of neuronal cell death, radial migration, neurite formation, metabolism regulation, and behavioral control. Twelve MAPKKKs, two MAPKs, and three JNKs can be combined in triads with specific scaffold proteins to form a large array of JNK signaling modules. These various modules are believed to facilitate JNK participation in specific biological functions. Indeed, the difference in phenotypes displayed by $M k k 4^{f l o x} / f l o x$ Nestin-Cre and Mkk7flox/flox Nestin-Cre mice reinforces the notion that there are distinct JNK signaling modules that may be MKK4 dependent or MKK7 dependent. Thus, to truly elucidate the JNK signaling network, it will be necessary to investigate JNK signaling at every step of its hierarchy: MAPKs, MAPKKs, and MAPKKKs.

The brain contains many different cell types, including various classes of neurons, astrocytes, and oligodendrocytes. To date, cell type-specific analyses of JNK signaling have not been performed. Future reviews will no doubt focus on the use of cell type-specific cKO mice to further dissect the striking reach of JNK signaling in normal and abnormal physiology.

\section{Acknowledgments}

This work was supported by grants from the Ministry of Education, Culture, Sports, Science and Technology of Japan, the Ministry of Health, Labour and Welfare of Japan, and the Japan Society for the Promotion of Science.

\section{References}

[1] R. J. Davis, "Signal transduction by the JNK group of MAP kinases," Cell, vol. 103, no. 2, pp. 239-252, 2000.

[2] L. Chang and M. Karin, "Mammalian MAP kinase signalling cascades," Nature, vol. 410, no. 6824, pp. 37-40, 2001.

[3] M. Hibi, A. Lin, T. Smeal, A. Minden, and M. Karin, "Identification of an oncoprotein- and UV-responsive protein kinase that binds and potentiates the c-Jun activation domain," Genes and Development, vol. 7, no. 11, pp. 2135-2148, 1993.

[4] A. Gdalyahu, I. Ghosh, T. Levy et al., "DCX, a new mediator of the JNK pathway," EMBO Journal, vol. 23, no. 4, pp. 823-832, 2004.

[5] T. Kawauchi, K. Chihama, Y. I. Nabeshima, and M. Hoshino, "The in vivo roles of STEF/Tiam1, Rac1 and JNK in cortical neuronal migration," EMBO Journal, vol. 22, no. 16, pp. 41904201, 2003.

[6] T. Tararuk, N. Östman, W. Li et al., "JNK1 phosphorylation of SCG10 determines microtubule dynamics and axodendritic length," Journal of Cell Biology, vol. 173, no. 2, pp. 265-277, 2006.

[7] M. A. Bogoyevitch and B. Kobe, "Uses for JNK: the many and varied substrates of the c-Jun N-terminal kinases,"
Microbiology and Molecular Biology Reviews, vol. 70, no. 4, pp. 1061-1095, 2006.

[8] X. Wang, A. Destrument, and C. Tournier, "Physiological roles of MKK4 and MKK7: insights from animal models," Biochimica et Biophysica Acta, vol. 1773, no. 8, pp. 1349-1357, 2007.

[9] Y. Asaoka and H. Nishina, "Diverse physiological functions of MKK4 and MKK7 during early embryogenesis," Journal of Biochemistry, vol. 148, no. 4, pp. 393-401, 2010.

[10] W. Haeusgen, T. Herdegen, and V. Waetzig, "The bottleneck of JNK signaling: molecular and functional characteristics of MKK4 and MKK7," European Journal of Cell Biology, vol. 90, no. 6-7, pp. 536-544, 2011.

[11] S. Lawler, Y. Fleming, M. Goedert, and P. Cohen, "Synergistic activation of SAPK1/JNK1 by two MAP kinase kinases in vitro," Current Biology, vol. 8, no. 25, pp. 1387-1390, 1998.

[12] H. Kishimoto, K. Nakagawa, T. Watanabe et al., "Different properties of SEK1 and MKK7 in dual phosphorylation of stress-induced activated protein kinase SAPK/JNK in embryonic stem cells," Journal of Biological Chemistry, vol. 278, no. 19, pp. 16595-16601, 2003.

[13] B. D. Cuevas, A. N. Abell, and G. L. Johnson, "Role of mitogenactivated protein kinase kinase kinases in signal integration," Oncogene, vol. 26, no. 22, pp. 3159-3171, 2007.

[14] M. Dickens, J. S. Rogers, J. Cavanagh et al., "A cytoplasmic inhibitor of the JNK signal transduction pathway," Science, vol. 277, no. 5326, pp. 693-696, 1997.

[15] J. Yasuda, A. J. Whitmarsh, J. Cavanagh, M. Sharma, and R. J. Davis, "The JIP group of mitogen-activated protein kinase scaffold proteins," Molecular and Cellular Biology, vol. 19, no. 10, pp. 7245-7254, 1999.

[16] M. Ito, K. Yoshioka, M. Akechi et al., "JSAP1, a novel jun Nterminal protein kinase (JNK)-binding protein that functions as a scaffold factor in the JNK signaling pathway," Molecular and Cellular Biology, vol. 19, no. 11, pp. 7539-7548, 1999.

[17] N. Kelkar, S. Gupta, M. Dickens, and R. J. Davis, "Interaction of a mitogen-activated protein kinase signaling module with the neuronal protein JIP3," Molecular and Cellular Biology, vol. 20, no. 3, pp. 1030-1043, 2000.

[18] C. Y. Kuan, D. D. Yang, D. R. Samanta Roy, R. J. Davis, P. Rakic, and R. A. Flavell, "The Jnk1 and Jnk2 protein kinases are required for regional specific apoptosis during early brain development," Neuron, vol. 22, no. 4, pp. 667-676, 1999.

[19] K. Sabapathy, W. Jochum, K. Hochedlinger, L. Chang, M. Karin, and E. F. Wagner, "Defective neural tube morphogenesis and altered apoptosis in the absence of both JNK1 and JNK2," Mechanisms of Development, vol. 89, no. 1-2, pp. 115124, 1999.

[20] N. Westerlund, J. Zdrojewska, A. Padzik et al., "Phosphorylation of SCG10/stathmin-2 determines multipolar stage exit and neuronal migration rate," Nature Neuroscience, vol. 14, no. 3, pp. 305-313, 2011.

[21] B. Björkblom, N. Östman, V. Hongisto et al., "Constitutively active cytoplasmic c-Jun N-terminal kinase 1 is a dominant regulator of dendritic architecture: role of microtubuleassociated protein 2 as an effector," Journal of Neuroscience, vol. 25, no. 27, pp. 6350-6361, 2005.

[22] L. Chang, Y. Jones, M. H. Ellisman, L. S. B. Goldstein, and M. Karin, "JNK1 is required for maintenance of neuronal microtubules and controls phosphorylation of microtubuleassociated proteins," Developmental Cell, vol. 4, no. 4, pp. 521533, 2003.

[23] W. Dauer and S. Przedborski, "Parkinson's disease: mechanisms and models," Neuron, vol. 39, no. 6, pp. 889-909, 2003. 
[24] S. Hunot, M. Vila, P. Teismann et al., "JNK-mediated induction of cyclooxygenase 2 is required for neurodegeneration in a mouse model of Parkinson's disease," Proceedings of the National Academy of Sciences of the United States of America, vol. 101, no. 2, pp. 665-670, 2004.

[25] D. D. Yang, C. Y. Kuan, A. J. Whitmarsh et al., "Absence of excitotoxicity-induced apoptosis in the hippocampus of mice lacking the Jnk3 gene," Nature, vol. 389, no. 6653, pp. 865-870, 1997.

[26] C. Y. Kuan, A. J. Whitmarsh, D. D. Yang et al., "A critical role of neural-specific JNK3 for ischemic apoptosis," Proceedings of the National Academy of Sciences of the United States of America, vol. 100, no. 25, pp. 15184-15189, 2003.

[27] G. Pirianov, K. G. Brywe, C. Mallard et al., "Deletion of the c-Jun N-terminal kinase 3 gene protects neonatal mice against cerebral hypoxic-ischaemic injury," Journal of Cerebral Blood Flow and Metabolism, vol. 27, no. 5, pp. 1022-1032, 2007.

[28] Y. Morishima, Y. Gotoh, J. Zieg et al., " $\beta$-amyloid induces neuronal apoptosis via a mechanism that involves the c-Jun N-terminal kinase pathway and the induction of fas ligand," Journal of Neuroscience, vol. 21, no. 19, pp. 7551-7560, 2001.

[29] S. Brecht, R. Kirchhof, A. Chromik et al., "Specific pathophysiological functions of JNK isoforms in the brain," European Journal of Neuroscience, vol. 21, no. 2, pp. 363-377, 2005.

[30] B. F. Belgardt, J. Mauer, F. T. Wunderlich et al., "Hypothalamic and pituitary c-Jun $\mathrm{N}$-terminal kinase 1 signaling coordinately regulates glucose metabolism," Proceedings of the National Academy of Sciences of the United States of America, vol. 107, no. 13, pp. 6028-6033, 2010.

[31] E. K. Unger, M. L. Piper, L. E. Olofsson, and A. W. $\mathrm{Xu}$, "Functional role of c-Jun-N-terminal kinase in feeding regulation," Endocrinology, vol. 151, no. 2, pp. 671-682, 2010.

[32] G. Tuncman, J. Hirosumi, G. Solinas, L. Chang, M. Karin, and G. S. Hotamisligil, "Functional in vivo interactions between JNK1 and JNK2 isoforms in obesity and insulin resistance," Proceedings of the National Academy of Sciences of the United States of America, vol. 103, no. 28, pp. 10741-10746, 2006.

[33] J. Hirosumi, G. Tuncman, L. Chang et al., "A central, role for JNK in obesity and insulin resistance," Nature, vol. 420, no. 6913, pp. 333-336, 2002.

[34] H. Nishina, C. Vaz, P. Billia et al., "Defective liver formation and liver cell apoptosis in mice lacking the stress signaling kinase SEK1/MKK4," Development, vol. 126, no. 3, pp. 505516, 1999.

[35] T. Watanabe, K. Nakagawa, S. Ohata et al., "SEK1/MKK4mediated SAPK/JNK signaling participates in embryonic hepatoblast proliferation via a pathway different from NF- $\kappa \mathrm{B}$ induced anti-apoptosis," Developmental Biology, vol. 250, no. 2, pp. 332-347, 2002.

[36] T. Wada, N. Joza, H. M. Cheng et al., "MKK7 couples stress signalling to $\mathrm{G} 2 / \mathrm{M}$ cell-cycle progression and cellular senescence," Nature Cell Biology, vol. 6, no. 3, pp. 215-226, 2004.

[37] X. Wang, B. Nadarajah, A. C. Robinson et al., "Targeted deletion of the mitogen-activated protein kinase kinase 4 gene in the nervous system causes severe brain developmental defects and premature death," Molecular and Cellular Biology, vol. 27, no. 22, pp. 7935-7946, 2007.

[38] T. Yamasaki et al., "Stress-activated protein kinase MKK7 regulates axon elongation in the developing cerebral cortex," The Journal of Neuroscience, vol. 31, no. 46, pp. 16872-16883, 2011.
[39] D. Horiuchi, C. A. Collins, P. Bhat, R. V. Barkus, A. DiAntonio, and W. M. Saxton, "Control of a kinesin-cargo linkage mechanism by JNK pathway kinases," Current Biology, vol. 17, no. 15, pp. 1313-1317, 2007.

[40] S. I. Hirai, F. C. De, T. Miyata et al., "The c-Jun N-terminal kinase activator dual leucine zipper kinase regulates axon growth and neuronal migration in the developing cerebral cortex," Journal of Neuroscience, vol. 26, no. 46, pp. 1199212002, 2006.

[41] S.-I. Hirai, Y. Banba, T. Satake, and S. Ohno, "Axon formation in neocortical neurons depends on stage-specific regulation of microtubule stability by the dual leucine zipper kinase-c-jun N-terminal kinase pathway," Journal of Neuroscience, vol. 31, no. 17, pp. 6468-6480, 2011.

[42] H. Ichijo, E. Nishida, K. Irie et al., "Induction of apoptosis by ASK1, a mammalian MAPKKK that activates SAPK/JNK and p38 signaling pathways," Science, vol. 275, no. 5296, pp. 90-94, 1997.

[43] K. Tobiume, A. Matsuzawa, T. Takahashi et al., "ASK1 is required for sustained activations of JNK/p38 MAP kinases and apoptosis," EMBO Reports, vol. 2, no. 3, pp. 222-228, 2001.

[44] C. Harada, K. Nakamura, K. Namekata et al., "Role of apoptosis signal-regulating kinase 1 in stress-induced neural cell apoptosis in vivo," American Journal of Pathology, vol. 168, no. 1, pp. 261-269, 2006.

[45] H. Nishitoh, A. Matsuzawa, K. Tobiume et al., "ASK1 is essential for endoplasmic reticulum stress-induced neuronal cell death triggered by expanded polyglutamine repeats," Genes and Development, vol. 16, no. 11, pp. 1345-1355, 2002.

[46] H. Nishitoh, H. Kadowaki, A. Nagai et al., "ALS-linked mutant SOD1 induces ER stress- and ASK1-dependent motor neuron death by targeting Derlin-1," Genes and Development, vol. 22, no. 11, pp. 1451-1464, 2008.

[47] K. Kumakura, H. Nomura, T. Toyoda et al., "Hyperactivity in novel environment with increased dopamine and impaired novelty preference in apoptosis signal-regulating kinase 1 (ASK1)-deficient mice," Neuroscience Research, vol. 66, no. 3, pp. 313-320, 2010.

[48] B. Glise, H. Bourbon, and S. Noselli, "hemipterous encodes a novel Drosophila MAP kinase kinase, required for epithelial cell sheet movement," Cell, vol. 83, no. 3, pp. 451-461, 1995.

[49] J. R. Riesgo-Escovar, M. Jenni, A. Fritz, and E. Hafen, "The Drosophila jun-N-terminal kinase is required for cell morphogenesis but not for DJun-dependent cell fate specification in the eye," Genes and Development, vol. 10, no. 21, pp. 27592768, 1996.

[50] H. K. Sluss, Z. Han, T. Barrett, R. J. Davis, and Y. T. Ip, "A JNK signal transduction pathway that mediates morphogenesis and an immune response in Drosophila," Genes and Development, vol. 10, no. 21, pp. 2745-2758, 1996.

[51] H. Taru, K. I. Iijima, M. Hase, Y. Kirino, Y. Yagi, and T. Suzuki, "Interaction of Alzheimer's $\beta$-amyloid precursor family proteins with scaffold proteins of the JNK signaling cascade," Journal of Biological Chemistry, vol. 277, no. 22, pp. 20070-20078, 2002.

[52] G. A. Morfini, Y. M. You, S. L. Pollema et al., "Pathogenic huntingtin inhibits fast axonal transport by activating JNK3 and phosphorylating kinesin," Nature Neuroscience, vol. 12, no. 7, pp. 864-871, 2009.

[53] P. Xu, M. Das, J. Reilly, and R. J. Davis, "JNK regulates FoxOdependent autophagy in neurons," Genes and Development, vol. 25 , no. 4, pp. 310-322, 2011. 
[54] L. Yu, A. Alva, H. Su et al., "Regulation of an ATG7-beclin 1 program of autophaglic cell death by caspase-8," Science, vol. 304, no. 5676, pp. 1500-1502, 2004.

[55] S. Shimizu, A. Konishi, Y. Nishida et al., "Involvement of JNK in the regulation of autophagic cell death," Oncogene, vol. 29, no. 14, pp. 2070-2082, 2010. 

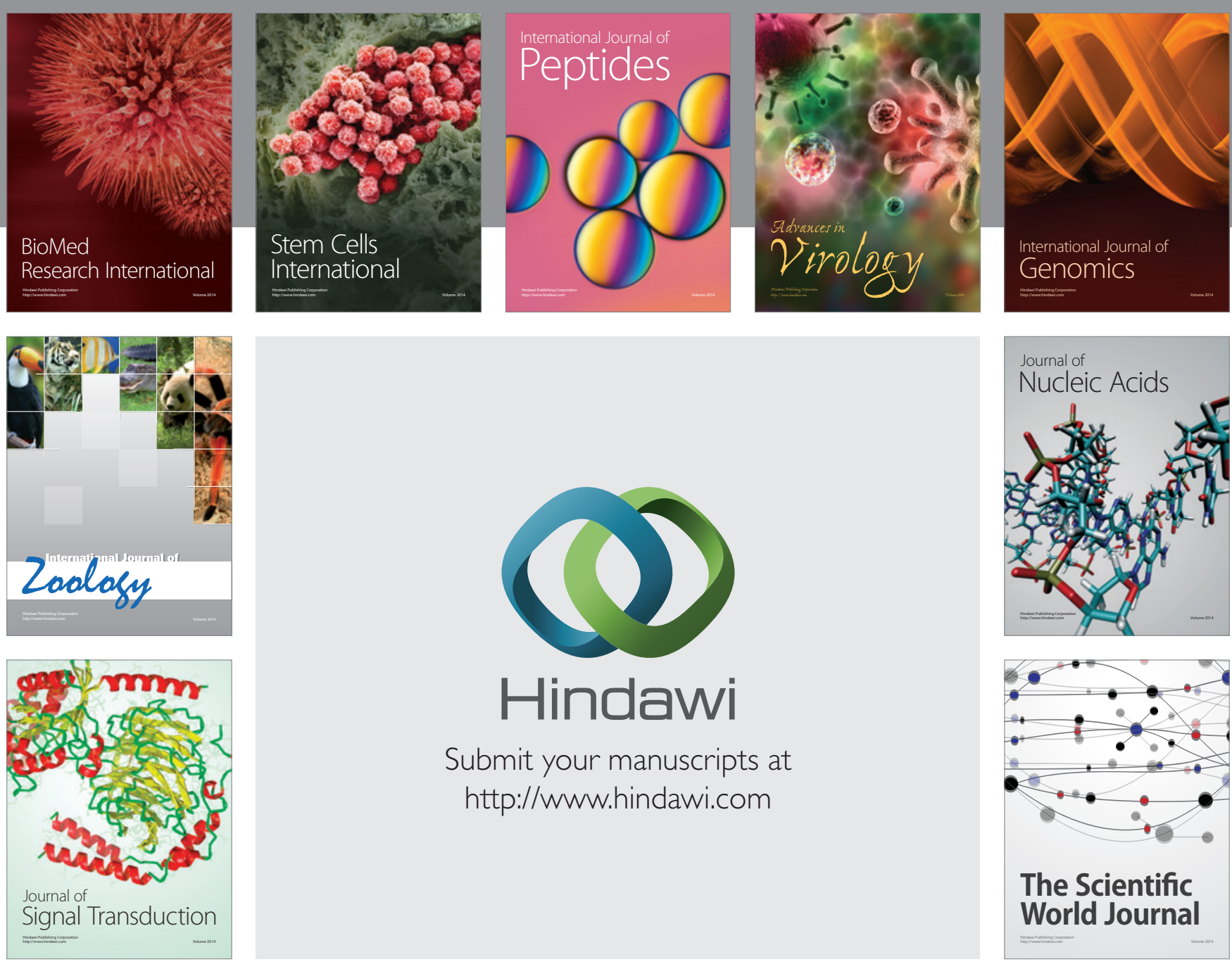

Submit your manuscripts at

http://www.hindawi.com
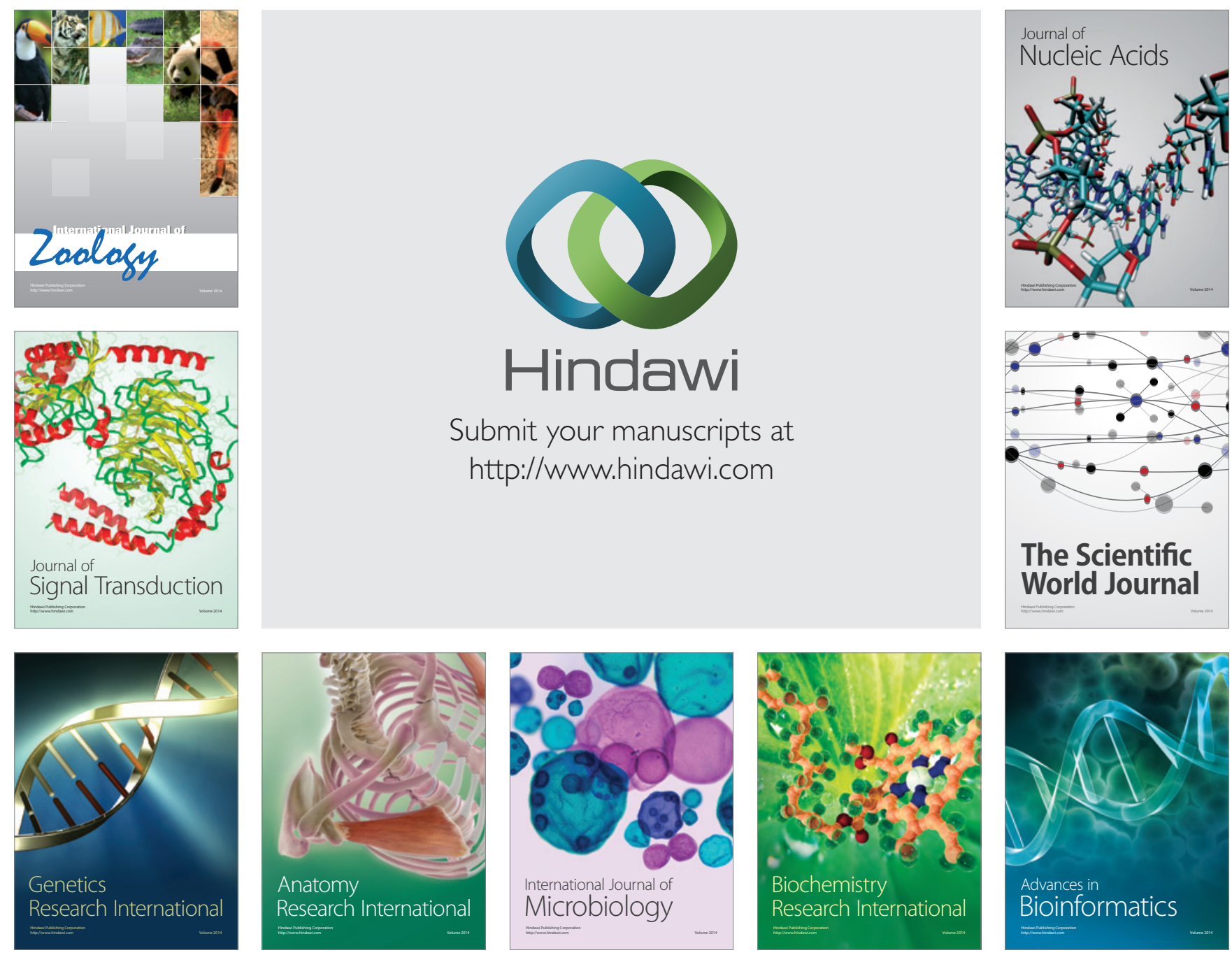

The Scientific World Journal
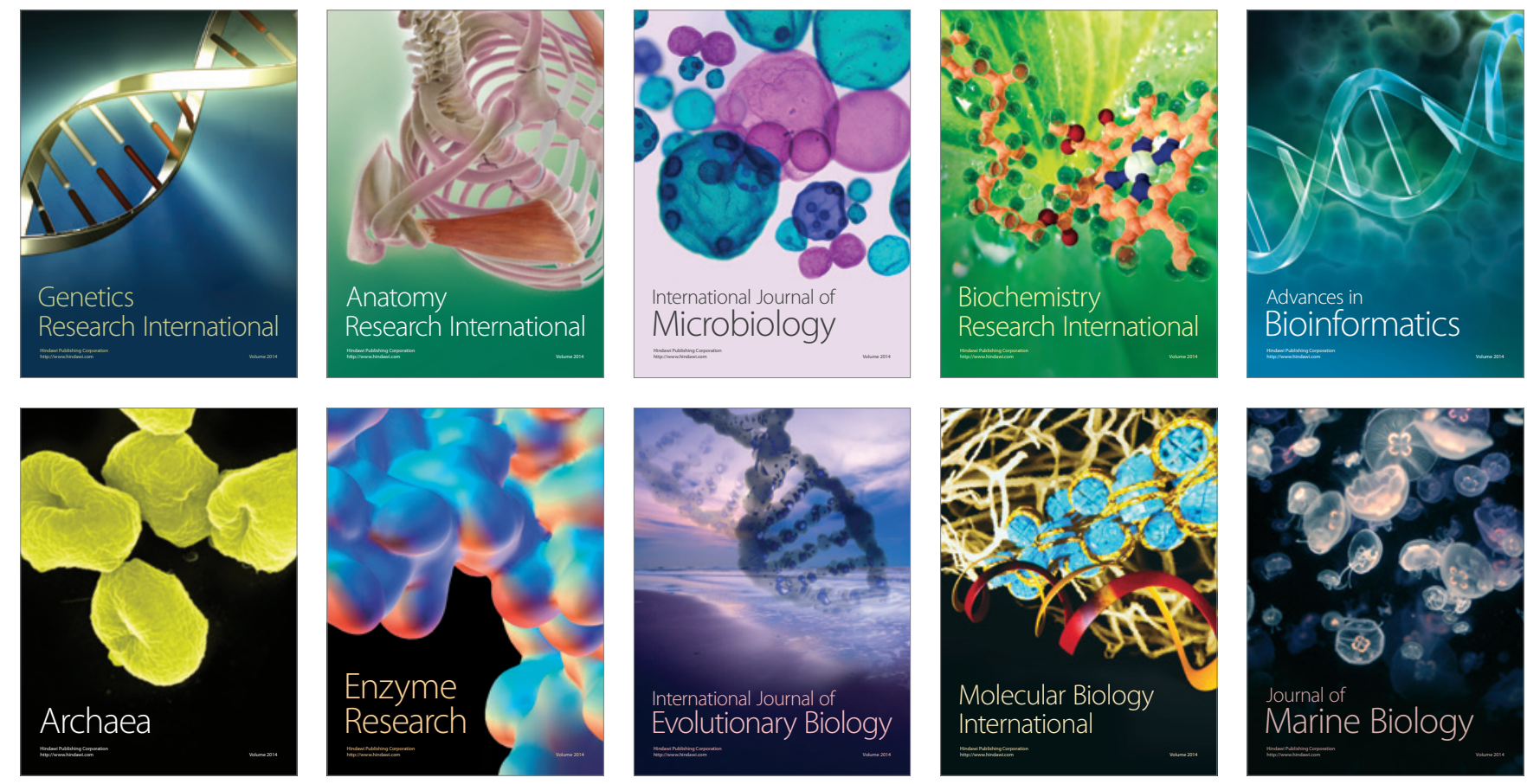